\title{
Visions for Mathematical Learning: The Inspirational Legacy of Seymour Papert (1928-2016)
}

Celia Hoyles and Richard Noss (UCL Knowledge Lab, University College, London, UK)

Seymour Papert, who died on 31 July, was a mathematician with two PhDs in pure mathematics, from the University of Witwatersrand, South Africa, and the University of Cambridge, UK. He was a founder of artificial intelligence with Marvin Minsky at MIT, a psychologist working alongside Jean Piaget, a political activist against apartheid and, on a personal level, a wonderful cook and loyal friend. Since his death, the web has been awash with reminiscences and detailed accounts of his intellectual contributions, not only to the fundamental subjects in which he was the undisputed leader but also to the field of education, to a scholar who believed and showed that the computer, or at least the very carefully crafted use of the computer, could introduce young and old alike to the joys and power of mathematics and mathematical thinking.

In this short article, we have selected four pieces of work that directly impacted on the mathematics education field and community. Significantly, these are among his less well-known lectures and papers and we hope that, by airing them, the realisation of Papert's vision of a new kind of learnable mathematics may be one step closer.

\section{0: Keynote in ICME Berkeley, USA}

Seymour gave one of the four plenaries at ICME 1980. Sadly, as far as we can tell, there was no transcript produced of Seymour's remarks. We are, however, grateful to Jeremy Kilpatrick (who attended the talk) for pointing us to a 1980 book edited by Lynn Steen and Don Albers, which includes a 4-page synopsis of Seymour's talk. ${ }^{1}$

Apparently, Seymour was inspirational. From the abstract, we know that he began:

"We are at the beginning of what is the decade of mathematics education. Not just in how children learn, but what they learn: we will see dramatic changes in what children learn; we will see subject matters that formerly seemed inaccessible or difficult even at college level learned by young children; we will see changes in where learning takes place, and in the process of learning itself."

\footnotetext{
1 https://books.google.cz/books?id=zcq9BwAAQBAJ\&pg=P $\mathrm{A} 12 \& \operatorname{lpg}=\mathrm{PA} 12 \& \mathrm{dq}=\% 22$.
} 


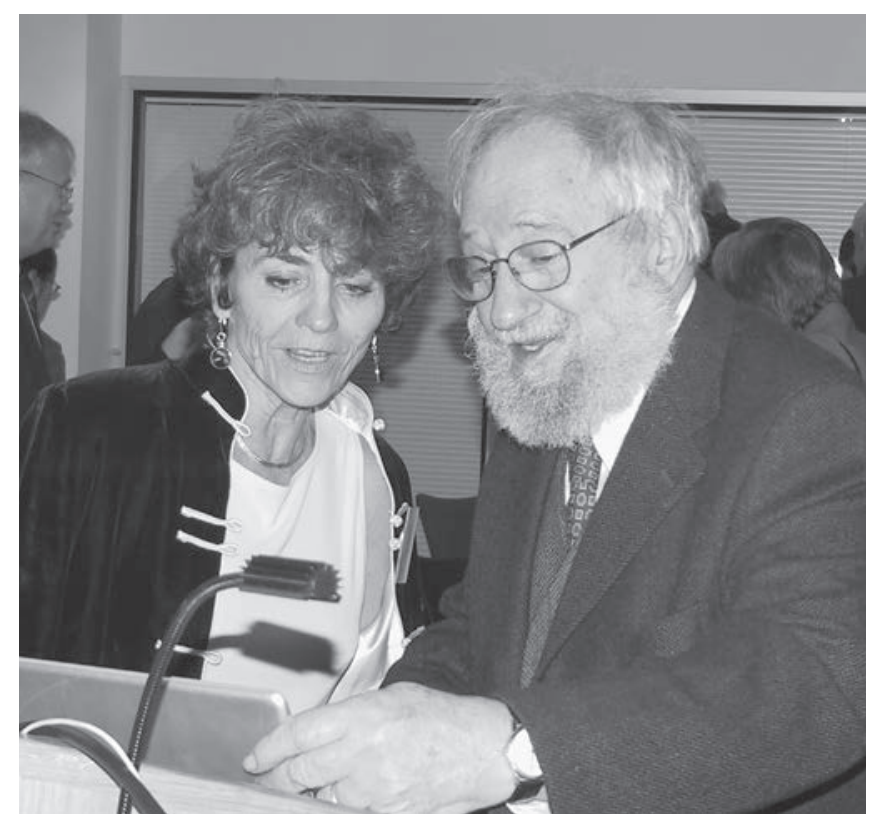

Seymour Papert and Celia Hoyles.

Even at this early stage, some 30 years before the presence of computers in school became commonplace, Seymour was addressing the question of epistemology, the 'what' of mathematics education - a theme that permeated his writings and speeches ever since.

\section{6: Keynote at the Tenth Conference of the International Group for the Psychology of Mathematics Education (PME 10) in London, UK}

The title of Seymour's talk was "Beyond the Cognitive: the Other Face of Mathematics". ${ }^{2}$ This talk was again inspirational and maybe a little controversial. He began by stating how he

"shared with Piaget the heuristic value that trying as hard as one can to understand as much as one can of children's mathematics and mathematicians' mathematics in the same categories. Doing so can illuminate both sides" (p. 1).

How right he was - as so many of us have now experienced in our own work. Seymour argued for a greater importance to be accorded to the affective side of mathematics; remember that this keynote was 30 years ago, when mathematics education research was firmly grounded in the cognitive paradigm. In particular, Seymour noted how some people tended to identify with mathematical objects: a precursor of the hugely influential movement 'embodied mathematics'? "Do you observe the mathematical scene in your head or are you in it?" he asks (p. 2). And then the punchline that we will never forget: he showed how the "Euclidean propositions can be seen in a different light as special cases of turtle theorems" (p. 3), thus illustrating beautifully how a geometry that

\footnotetext{
2 http://dailypapert.com/wp-content/uploads/2015/07/BeyondTheCognitive.pdf
}

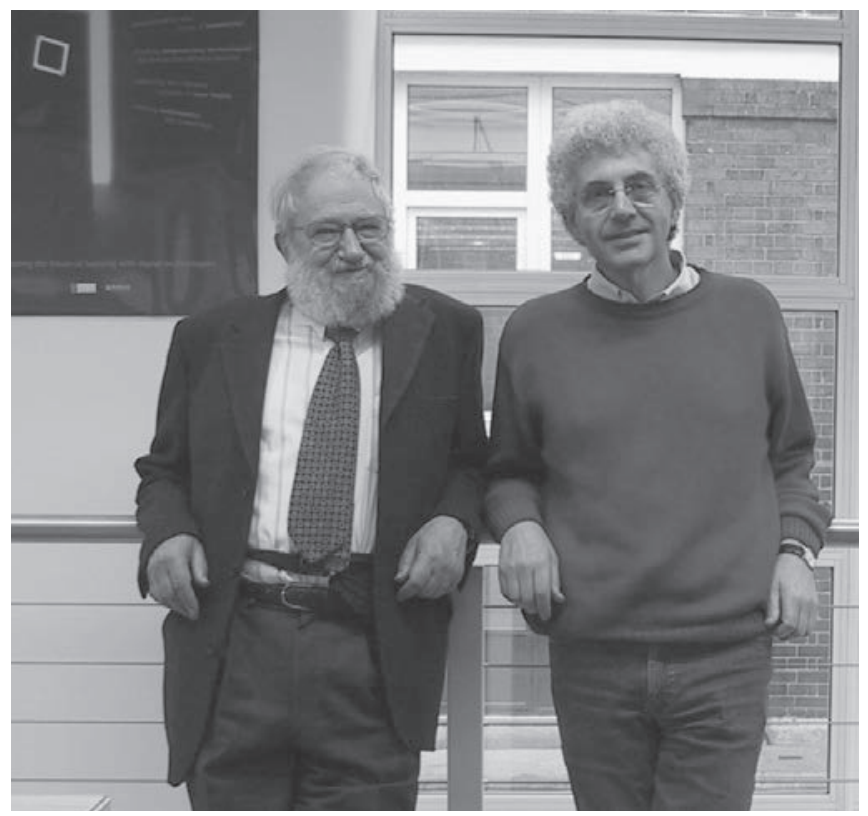

Seymour Papert and Richard Noss.

starts with the intuitions of body movement rather than abstract points and lines can be no less rigorous but considerably more inviting.

\section{6: Launching a new journal: the International Journal of Computers for Mathematics Learning (IJCML)}

In 1996, Seymour became the founding editor of a new journal, IJCML. In the first issue, he undertakes "An Exploration in the Space of Mathematics Educations". This brilliant contribution begins memorably:

A mathematical metaphor frames the intentions of this paper. Imagine that we know how to construct an $N$-dimensional space, $M E$, in which each point represents an alternative mathematics education - or ame - and each dimension a feature, such as a component of content, a pedagogical method, a theoretical or ideological position. Each "reform" of mathematics education introduces new points and each fundamental idea a new dimension. Thus, if one considers a particular point (an ame) in ME, among its many "coordinates" are a (metaphorical) measure that runs from informal to formal and another that runs from instructionist to constructivist. In the paper I shall define seven more such oppositional principles that have not been recognized in the past as structuring choices in mathematics education. (Papert, 1996)

The reader will not miss the daring and imaginative style of this metaphor. The article focuses on how the medium of expression can make any specific 'ame' seem 'natural', again using elementary geometric examples as illustration. But Seymour argues:

"...there is no doubt that in general much more can be done at an elementary level with dynamic than with algebraic characterizations of curves". 
Recall that this was a decade or so before dynamic geometry became widespread! In 2004, Seymour took up the theme of the mediation of knowledge more generally in a little-known but, for us, highly significant speech to open the London Knowledge Lab, where we both work. Take a look at https://mediacentral.ucl.ac.uk/Play/3004.

\section{6: Opening keynote to ICMI 17 Study Conference, Technology Revisited, Vietnam}

In July 2002, the ICMI Executive Committee launched the 17th ICMI Study, called "Technology Revisited", the title reflecting the fact that the very first ICMI Study, held in Strasbourg in 1985, focused on the influence of computers and informatics on mathematics and its teaching. The Programme Committee wanted the Study Conference to be opened by a scholar with vision, experience and stature in the fields of mathematics, mathematics education and technology. We chose Seymour and to our delight he accepted by return of email. The tone of his emails became more and more excited as the conference approached. In his talk, Seymour spoke to the title '30 Years of Digital Technologies in Mathematics Education and the Future', using the recently prototyped and revolutionary '100 dollar laptop' (renamed the 'XO') to present his talk. He argued that, with full and easy access to computers, we face the challenge to consider not only how existing knowledge can be addressed in technologyenhanced ways but also that we should reserve at least $10 \%$ of our time and energy to consider what new types of mathematical knowledge and practices might emerge as a result. His accident the next day was a most terrible shock to us and to all the participants, and the conference struggled to continue after this tragedy, even as Seymour struggled in hospital. The best tribute we could think of was to try to keep the spirit of his ambition alive throughout the meeting by asking for participants to consider 'Seymour's $10 \%$ ' in all their sessions and their subsequent papers. (Adapted from Hoyles, C., \& Lagrange, J. B., 2010)

We hope that this short piece will keep Seymour's vision and struggle alive.

\section{Acknowledgment}

This article was originally published in the November 2016 issue of the ICMI Newsletter (http://www.mathunion.org/ fileadmin/ICMI/files/News/ICMI_Newsletter_November_2016_LK_final.pdf). We gratefully acknowledge its editors Abraham Arcavi (ICMI Secretary General) and Cheryl E. Praeger (ICMI Vice-president) for the reprint permission.

\section{References}

Hoyles, C., \& Lagrange, J.-B. (Eds) (2010) Mathematics Education and Technology - Rethinking the Terrain, Springer.

Steen, L., \& Albers, D. J. (Eds) (1981) Teaching Teachers, Teaching Students - Reflections on Mathematical Education, Springer.

Papert, S., (1996) An Exploration in the Space of Mathematics Educations, Vol. 1, No. 1, pp. 95-123.

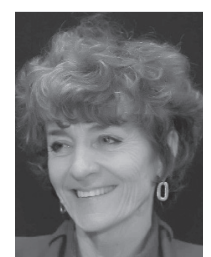

Celia Hoyles has been a professor of mathematics education at the Institute of Education, University of London, since 1986. She was a recipient of the ICMI Hans Freudenthal Medal in 2004 and the Royal Society Kavli Education Medal in 2011. She was President of the Institute of Mathematics and its Applications in 2014-15 and a Member of the European Mathematical Society (EMS) Committee for Mathematics Education in 2008-16.

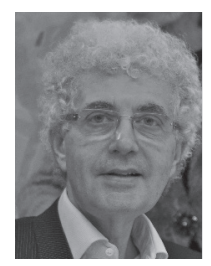

Richard Noss is a founding member of the Logo and Mathematics Education Group inspired by Seymour. He became a professor of mathematics education at the Institute of Education in 1996. He was the founder and director of the London Knowledge Lab - exploring the future of learning with digital technologies - for its first decade. Richard co-authored (with Celia Hoyles) 'Windows on Mathematical Meanings: Learning Cultures and Computers' in 1996. 